\title{
Tumor Suppressor Genes: A Key to the Cancer Puzzle?
}

\author{
Steven B. Oppenheimer
}

$\mathrm{T}$ HE molecular basis of cancer causation, to this date, is still poorly understood. Although numerous carcinogens have been identified (review in Oppenheimer 1985; 1987a; 1987b), the mechanism(s) of their action remains uncertain. In recent years indirect evidence has accumulated that suggested that these carcinogens act at the DNA level, altering or activating certain genes to form oncogenes (cancer causing genes), that can produce protein products that lead to the malignant transformation of cells (Oppenheimer 1985; 1987a).

Oncogenes have been identified in cancer cells and can cause some cells to display malignant properties in culture and when transplanted into organisms (review in Oppenheimer 1985; 1987a). No direct proof exists, however, that oncogenes cause most human cancers. As the excitement about oncogenes became tempered from the realization that direct proof for this link was slow in coming, another group of genes has captured the attention of the scientific community. These genes termed tumor suppressor genes (or antioncogenes or anticancer genes) have been, in a growing number of cases, directly linked to preventing human cancer, and in their absence, causing it (Sager 1989). The cancer puzzle is beginning to become less confusing. As this review will show, many human cancers may be caused by carcinogens directly destroying or damaging tumor suppressor genes and possibly also by activating oncogenes. This author feels that the data that will be presented are already sufficient to conclude that the discovery of tumor suppressor genes might become one of the most important true "breakthroughs" in solving the cancer puzzle. We can leave it to our students to research, debate and further discuss this intriguing work in our classes.

The beginning of the tumor suppressor gene story perhaps begins with work by Henry Harris and colleagues in 1969 who showed that malignancy was suppressed when highly malignant mouse Ehrlich, SEWA and MSWBS ascites tumor cells and more

Steven B. Oppenheimer is professor of biology and director of the Center for Cancer and Developmental Biology at California State University, Northridge, 1811 Nordhoff St., Northridge, CA 91330. normal A9 mouse fibroblast cells were fused in culture. Since the complete genetic complements of both cell types remained intact in the newly created fusion product, the results suggested the existence of some sort of tumor suppressor gene, contributed by the A9 cell, that "normalized" the highly malignant cell (Harris et al. 1969). This suggestion was strengthened when it was found that tumor forming ability returned to the hybrid cells when chromosomes from the more normal parent cell were lost during propagation in culture (Harris et al. 1969; Harris 1986; Sager 1989).

The second important piece of the tumor suppressor gene story began with work by Alfred Knudson at the M.D. Anderson Cancer Center and Tumor Institute in Houston. Knudson examined the family histories of 48 patients with a rare cancer called retinoblastoma. This is a cancer of the retinas of the eyes that usually afflicts infants and young children and strikes about one in 20,000 individuals. The cancer can be successfully treated if detected early, so many of these young patients grow up to have children who also often come down with the cancer at a rate as high as 50 percent. Knudson concluded from his analysis that the cancer must result from two mutations, the first probably inherited and the second occurring spontaneously after birth (Knudson 1971).

Enough evidence accumulated through the 1970s and early 1980s to prove Knudson correct.

Examination of retinoblastoma cells from patients showed that most had a specific region of both copies of chromosome 13 visibly missing (q14 region of chromosome 13) (Sager 1989). The gene responsible for preventing this cancer has been recently isolated in the q14 region of chromosome 13, based on its location on the chromosome, its absence in cell lines from patients with the cancer and its ability to suppress cancer when transferred into retinoblastoma cell lines (Sager 1989). All the results, taken together, provide rather definitive evidence that the absence of this gene (called RB) is the cause of retinoblastoma.

Individuals who develop familial retinoblastoma start life with one inherited defective RB gene (either mutated or deleted). This will not result in retinoblastoma, however, because the RB gene on the other copy of chromosome 13 provides enough gene prod- 
uct to prevent the cancer from developing. In the millions of retina cells of the eye, it is likely that a spontaneous mutation will occur in the RB gene of the normal copy of chromosome 13 in at least one cell. This cell then will have both RB genes missing or inactivated, causing the likely development of the cancer.

Two critical questions can now be asked. What protein does the RB gene encode? How does this protein in normal cells prevent the cancer from developing? Answers to these questions could quickly lead to ways of normalizing human cancer cells. A partial answer to these questions is available. The RB gene encodes a phosphorylated 110 kilodalton nuclear protein which has been proposed to act as an inhibitor of unregulated cell division. It is not yet known, however, how this protein maintains the non-cancerous state but it may be that RB is involved in essential steps in the differentiation of the normal retina. Lack of RB may cause cells to lose their differentiated characteristics, leading to reversion to a more embryonic, continuously dividing and aggressive state-that is, malignancy (Francke 1990).

Is there evidence that the absence of tumor suppressor genes is the cause of some of the more common cancers? Let us now turn to this intriguing story.

Loss of tumor suppressor genes has been implicated in causing numerous cancers including: melanoma, breast carcinoma, small cell lung cancer, cervical carcinoma, Wilms' tumor, osteosarcoma, stomach cancer, prostate cancer and colorectal cancer (Sager 1989; Stanbridge 1990; Solomon 1990; Bookstein et al. 1990; Hoffman 1989; Marx 1989).

Colorectal cancer is one of the most common malignancies in this nation. DNA was extracted from sections of frozen specimens of colorectal tumors. At least five different genetic changes have been identified in these cancer specimens, suggesting that this cancer may result from a multistep process. Many colon cancers appear to begin as areas of increased cell division, followed by development of more and more abnormal benign tumors called adenomas, which in turn lead to carcinomas that finally metastasize (Stanbridge 1990). Loss of a tumor suppressor gene from chromosome 5 seems responsible for initiating the process by transforming normal colon cells into cells that exhibit increased cell division. As the cells become more and more abnormal, tumor suppressor genes appear to be lost from chromosome 8 and 17. In addition, an oncogene called RAS is activated (Stanbridge 1990) (Figure 1).

A region on chromosome 18,18q21-22, was found to be the most commonly deleted region in colon cancers. A gene in this region known as DCC (deleted in colon cancer) is active in normal colon cells but usually not in colon cancer cells. This gene is a good candidate for a major colon cancer tumor suppressor

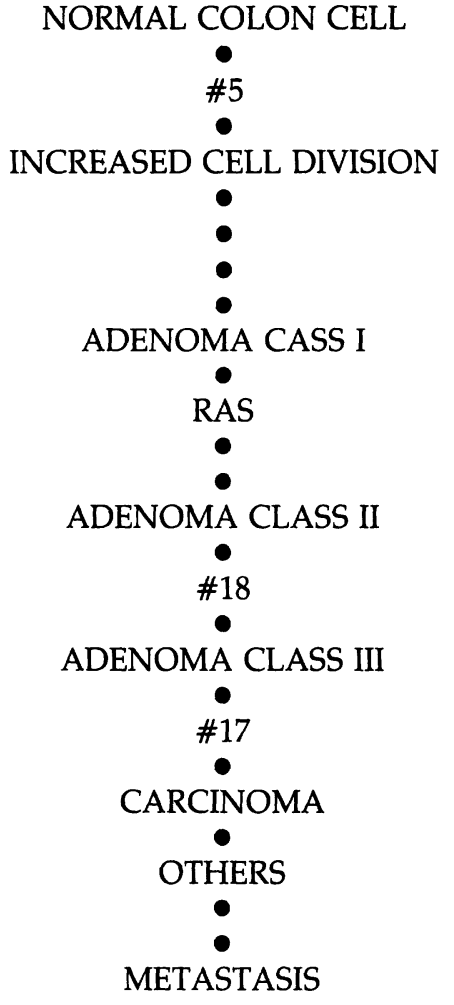

Figure 1. A Model for the Development of Colorectal Cancer. Numbers (\#) between steps denote the particular chromosome from which proposed tumor suppressor genes are lost or altered. RAS oncogene is also activated (Based on Stanbridge 1990).

gene. It is especially interesting that the product of DCC is similar to cell surface adhesion molecules (review in Oppenheimer 1985; Oppenheimer \& Lefevre 1989) and may prevent colon cancer by maintaining proper cell adhesion and growth control in the lining of the colon (Fearon et al. 1990, Solomon 1990) (Figure 1).

Small cell lung cancers are characterized by the presence of highly malignant small cells and are classified separately from non-small cell lung cancers. Both types of lung cancers show deletions or mutations in chromosomes 3,13 and 17. Interestingly, the retinoblastoma tumor suppressor gene was found to be deleted or rearranged in small cell lung cancer cells and RB messenger RNA was lost in these cells, but this was not found in a variety of non-small cell lung cancers (Marx 1989).

In order to definitively implicate the loss of a specific tumor suppressor gene in causing a cancer, experiments must be done to attempt to reverse the cancer by insertion of the tumor suppressor gene in question. This was recently accomplished with RB. Cloned RB DNA was introduced into a retinoblastoma cell line which had its own RB gene deleted and did not produce $\mathrm{RB}$ mRNA or RB protein. Insertion of the RB DNA into these cells caused reversion to cells 


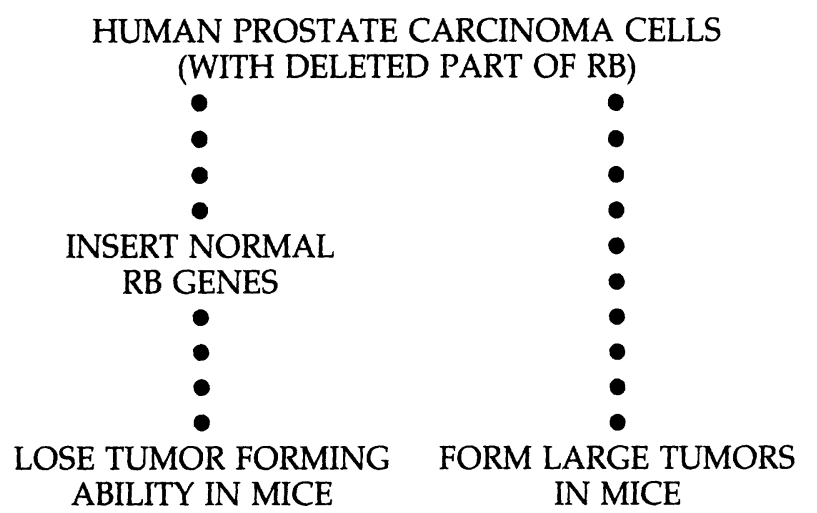

Figure 2. Loss of Tumor Forming Ability of Human Prostate Carcinoma Cells by Replacing a Mutated RB Gene (Based on Bookstein et al. 1990).

that grew normally in culture, expressed RB mRNA and $R B$ protein, and lost tumor-forming ability (Sager 1989). Reversal of malignancy was accomplished in a similar way with Wilms' tumor cells, a kidney cancer found in children (Weissman et al. 1987). Reversal of malignancy by insertion of a specific tumor suppressor gene into a cancer cell provides strong evidence that the gene in question prevents the cancer and that its loss causes it.

An exciting finding appeared in a recent issue of Science (Bookstein et al. 1990). Bookstein and colleagues at the University of California, San Diego, found that a human prostate carcinoma cell line contained RB gene protein that was abnormally short. The RB mRNA in these cells lacked 105 nucleotides encoded by exon 21-part of the RB gene. These prostate cancer cells formed large tumors when injected into experimental mice. When normal RB genes were inserted into these cells, they lost their ability to form tumors in mice (Figure 2). Immunological technologies might make it possible to target cancer cells around the body, carrying to them tumor suppressor genes. This approach, using gene therapy, could become an immensely important clinical anticancer strategy.

The demonstrated reversal of malignancy using tumor suppressor genes is convincing evidence that tumor suppressor genes may be among the most important developments in solving the cancer puzzle. Students in our classes can take up this issue in greater depth by library research, discussion and debate. A set of advances in cancer biology articles and enlarged figures is available free of charge by contacting the author.

\section{Acknowledgment}

This article is part of the Advances in Biological Science program, supported by the National Science Foundation, TPE Division, The Joseph Drown Foundation, The Thomas Eckstrom Trust and California State University, Northridge.

\section{References}

$\rightarrow$ Bookstein, R., Shew, J. Y., Chen, P. L., Scully, P. \& Lee, W. H. (1990). Suppression of tumorigenicity of human prostate carcinoma cells by replacing a mutated RB gene. Science, 247, 712-715.

$\rightarrow$ Fearon, E. R., Cho, K. R., Nigro, J. M., Kern, S. E., Simons, J. W., Ruppert, J. M., Hamilton, S. R., Preisinger, A. C., Thomas, G., Kinzler, K. W. \& Vogelstein, B. (1990). Identification of a chromosome $18 \mathrm{q}$ gene that is altered in colorectal cancers. Science, 247, 49-56.

$\rightarrow$ Francke, U. (1990). A gene for Wilms' tumor? Nature, 343, 692-694.

$\rightarrow$ Harris, H. (1986). Malignant tumors generated by recessive mutations. Nature, 323, 582-583.

$\rightarrow$ Harris, H., Miller, O. J., Klein, G., Worst, P. \& Tachibana, T. (1969). Suppression of malignancy by cell fusion. Nature, 223, 363-368.

$\rightarrow$ Hoffman, M. (1989). One Wilms' tumor gene is cloned; are there more? Science, 246, 1387.

$\rightarrow$ Knudson, A. G. (1971). Mutation and cancer: statistical study of retinoblastoma. Proceedings of the National Academy of Sciences, U.S.A., 68, 820-823.

$\rightarrow$ Marx, J. (1989). Many gene changes found in cancer. Science, 246, 1386-1388.

Oppenheimer, S. B. (1988). Antioncogenes, The Science Teacher, 55, 26-27.

Oppenheimer, S. B. (1985). Cancer: A Biological and Clinical Introduction (2nd ed). Boston: Jones and Bartlett.

$\rightarrow$ Oppenheimer, S. B. (1987a). Advances in cancer biology. The American Biology Teacher, 49, 11-15.

Oppenheimer, S. B. $(1987 \mathrm{~b})$. Ten strategies for cancer prevention. The Science Teacher, 54, 38-43.

Oppenheimer, S. B. \& Lefevre, G. (1989). Introduction to Embryonic Development ( $3 r d$ ed). Boston: Allyn and Bacon.

$\rightarrow$ Sager, R. (1989). Tumor suppressor genes: the puzzle and the promise. Science, 246, 1406-1412.

$\rightarrow$ Solomon, E. (1990). Colorectal cancer genes. Nature, 343, 412-414.

$\rightarrow$ Stanbridge, E. J. (1990). Identifying tumor suppressor genes in human colorectal cancer. Science, 247, 12-13.

$\rightarrow$ Weissman, B. E., Saxon, P. J., Pasquale, S. R., Jones, G. R., Geiser, A. G. \& Stanbridge, E. J. (1987). Introduction of a normal human chromosome 11 into a Wilms' tumor cell line controls its tumorigenic expression. Science, 236, 175-180. 